\title{
Aplicaciones relevantes en evaluación psicológica y psicometría: Editorial
}

\section{Relevant applications in psychological assessment and psychometrics: Editorial}

\author{
Eugenia V. Vinet ${ }^{\mathrm{a}}$ y Karina Rdz-Navarro ${ }^{\mathrm{b}}$ \\ Editoras invitadas \\ aUniversidad de La Frontera, Temuco, Cbile b Universidad Complutense de Madrid, Madrid, España
}

La investigación en ciencias del comportamiento y psicología implica necesariamente el desafío constante de la medición rigurosa de constructos de interés teórico abstracto y aplicado a la toma de decisiones y a la evaluación psicológica de casos particulares. En el número recién pasado de la Revista de Psicología (volumen 23, número 1) quisimos contribuir a la difusión de avances metodológicos en el área de la psicometría y la evaluación psicológica, invitando a investigadores nacionales y extranjeros a presentar sus estudios. El éxito de la convocatoria y la calidad de los trabajos recibidos nos permiten, con gran orgullo, presentarles este segundo número para el cual hemos seleccionado seis trabajos que, mediante una rigurosa aplicación de metodologías avanzadas de análisis, abordan diferentes ámbitos de la investigación en evaluación psicológica aplicados a la toma de decisiones en psicología clínica, educacional y social.

En este número los lectores podrán informarse sobre diversos avances en evaluación psicológica que cubren una amplia variedad de poblaciones (e.g., niños, adolescentes, jóvenes, adultos) y temáticas (i.e., la evaluación de características personales normales, los trastornos psicopatológicos, las relaciones de pareja, la preocupación por el medio ambiente y el emprendimiento individual) que creemos capturan una porción importante de la diversidad de áreas de investigación y aplicación de la disciplina.

En el primer artículo de este número Rossi-Casé et al., entregan parámetros normativos actualizados para el test de Matrices Progresivas de Raven obtenidos en una amplia muestra de estudiantes de 13 a 18 años, de la ciudad argentina de La Plata. El estudio se enriquece al comparar sus resultados con respecto a normas previas y al evaluar el efecto Flynn que se ha producido en los últimos cincuenta años en el desempeño de los sujetos participantes.

Siguiendo en el ámbito cognitivo, Luque, Elósegui y Casquero realizan un análisis en profundidad de niños y niñas con Capacidad Intelectual Límite, considerando las funciones y pruebas agrupadas en la Memoria de Trabajo y Velocidad de Procesamiento del WISC-IV, con el objeto de entender mejor esta condición de difícil diagnóstico y abordaje en el ámbito educacional.

A continuación, Brown, Capella y Antivilo, con la intención de proporcionar a la comunidad psicológica un instrumento que facilite la detección de psicopatología infanto-juvenil, hacen un detallado análisis de las propiedades psicométricas de la versión para padres del Strengths and Difficulties Questionnaire (SDQ), utilizando una amplia muestra de figuras parentales de niños y niñas chilenos de 4 a 11 años.

\section{Cómo citar:}

Vinet, E. V. y Rdz-Navarro, K. (2014). Aplicaciones relevantes en evaluación psicológica y psicometría: Editorial. Revista de Psicología, 23(2), 1-2. http://dx.doi.org/10.5354/0719-0581.2014.36143 
En otro ámbito, Fernández, Pavez y Dufey realizan, desde la perspectiva de la psicología evolucionaria, un estudio del Inventario de Comportamientos de la Pareja que Provocan Celos (ICPC), intentando dimensionar un fenómeno de las relaciones de pareja que, aunque normal, puede alcanzar condiciones psicopatológicas y que ha sido escasamente estudiado relacionando la psicometría y la psicología evolucionaria.

Finalmente, se presentan dos artículos que utilizan redes bayesianas, también conocidas como redes probabilísticas causales, que permiten caracterizar y explicar aspectos complejos de la realidad psicosocial. Así, Sánchez, de la Garza-González y Rodríguez ejemplifican didácticamente las redes bayesianas como técnica de análisis y generación de conocimiento, y abordan diversos aspectos de la percepción y la conducta ambiental de un grupo de estudiantes universitarios mexicanos. En tanto, López y RuizRuano utilizan las redes bayesianas para investigar los determinantes del emprendimiento individual que dan lugar a la creación de empresas. Ellos muestran que esta técnica permitiría definir relaciones entre variables no previstas teóricamente, desarrollar y/o mejorar modelos teóricos y, en el caso concreto analizado, aportar a la orientación de potenciales emprendedores que aspiran a crear su propio negocio.

A través de este conjunto de artículos, confiamos que los lectores puedan formarse una opinión documentada respecto de la utilidad de emplear técnicas de análisis psicométrico riguroso para describir fenómenos donde las variables vinculadas a la evaluación psicológica y la psicometría son fundamentales. Esperamos que los artículos que hemos seleccionado para ambos números sean del interés de los lectores de la Revista de Psicología y, una vez más, agradecemos el gran número de artículos recepcionados para esta sección temática en la cual hemos tenido el honor de colaborar. 